Quantitative Analysis of Hydrogen Isotopes in the Metal Hydride of the Neutron

Tube Target
H. Bach
S. Black
W. Chamberlin
D. Holt
E. Newman
F. Steinkruger
C. Walthers 


\section{DISCLAIMERR}

Portions of this document may be illegible in electronic image products. Images are produced from the best available original document. 


\title{
QUANTITATIVE ANALYSIS OF HYDROGEN ISOTOPES IN THE METAL HYDRIDE OF THE NEUTRON TUBE TARGET
}

by

H. Bach, S. Black, W. Chamberlin, D. Holt, E. Newman, F. Steinkruger, and C. Walthers

\begin{abstract}
We describe an experimental system for the quantitative analyses of hydrogen isotopes in metal hydride targets. The experimental system consists of a target desorption inlet system (TDIS) and a high-resolution mass spectrometer. The TDIS has a unique design that provides complete desorption of the gas and rapid, direct, and accurate measurements of pressure, volume, and temperature of the total gas desorbed from the target. The mass spectrometer measures partial pressures of the hydrogen isotopes from which the hydrogen isotopic ratios can be calculated. We present the operational parameters for optimum performance of the TDIS and results obtained from gas analyses of erbium hydride targets.
\end{abstract}

\section{INTRODUCTION}

Hydrogen isotopes from the metal hydride target, as shown in Fig. 1, of the neutron tube are thermally desorbed and quantitatively measured by a pressure-volume-temperature (PVT) mass spectrometer. In the early designs of similar desorption inlet systems, ${ }^{1,2}$ hydrogen isotopes were desorbed from the metal hydride targets into an evacuated volume. However, because the inlet system had three different temperature zones, quantitative determination of the total gas desorbed using PVT mass spectrometer measurement was very difficult.

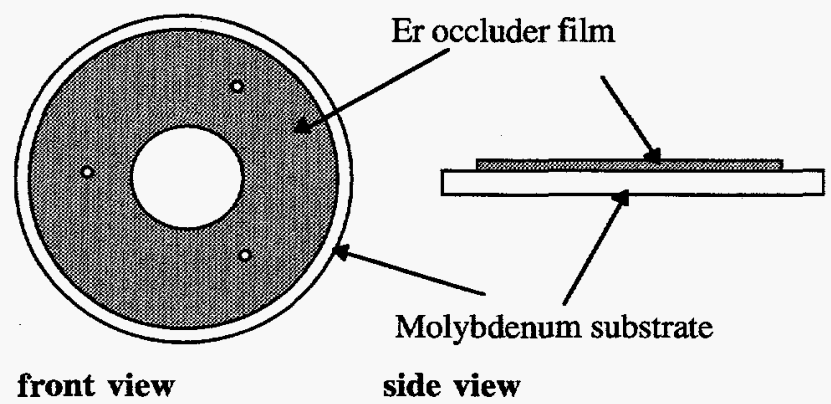

Fig. 1. Deuterated erbium target.
Furthermore, the desorption yield can never approach unity because even at high temperature and at equilibrium, there remains a finite fraction of hydrogen gas in the metal lattice. This fraction increases with a smaller volume inlet system.

To correct for the fraction of gas remaining in the target, an empirical correction factor was determined through a series of repeated pump-down measurements. In this technique, after a new equilibrium is reached, the system is cooled to room temperature and pumped out; then the desorption is repeated to reduce the total pressure and thus increase the amount of offgas. The total amount of hydrogen isotopes in each target is determined by summing up the amount of gases from all the repeated pump-down steps. The required number of repeated pump-down steps increases as the volume of the inlet system decreases. ${ }^{3}$ Because the amount of hydrogen isotopes varies as a result of the variation in metal film thickness and weight from production run to production run, the determination of the total hydrogen isotope could be subjected to a systematic error if the same correction factor were applied for all targets. For better accuracy, each target should have been evaluated independently without applying any correction. 
Therefore, our objectives are to simplify the system characterization, to desorb all the gas in one step, and to evaluate each target independently. We implemented an important modification to the inlet system as described below in which a turbomolecular pump is used to transfer the offgas into a storage volume and to maintain the system pressure at less than $10^{-5}$ torr. The optimum desorption parameters and total hydrogen isotopes are determined for both deuterated and tritiated erbium targets.

\section{EXPERIMENTAL SETUP}

\section{Model}

For a simple system, as illustrated in Fig. 2, the rate of desorption, $R_{d}$, is proportional to the deuterium concentration, $\mathrm{C}_{\mathrm{D} 2}$, in the target

$$
R_{d}=k_{d} C_{D_{2}},
$$

where $k_{d}$ is the desorption rate constant.

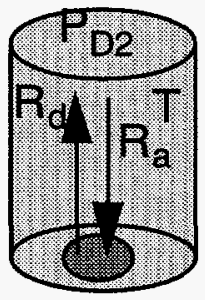

Fig. 2. A simple model of an unpumped system.

Using the same argument, the rate of absorption, $R_{a}$, is proportional to the partial pressure of deuterium, $\mathrm{P}_{\mathrm{D} 2}$,

$$
R_{a}=k_{a} P_{D_{2}},
$$

where $k_{a}$ is the absorption rate constant.

At equilibrium, the rates of desorption and absorption are equal; therefore, the concentration of deuterium in the target is proportional to the partial pressure of deuterium

$$
C_{D_{2}}=\frac{k_{a}}{k_{d}} P_{D_{2}} .
$$

If the system is modified with a turbomolecular pump to transfer the offgas to the gas storage vessel, as illustrated in Fig. 3, we could reduce the partial pressure of deuterium by a factor of $10^{5}$ based on the compression ratio of the turbomolecular pump for deuterium

$$
\frac{P_{1}}{P_{D 2}} \approx 10^{5} .
$$

Combining Eqs. (3) and (4), we have

$$
C_{D_{2}} \approx \frac{k_{a}}{k_{d}} \frac{P_{1}}{\left(10^{5}\right)}
$$

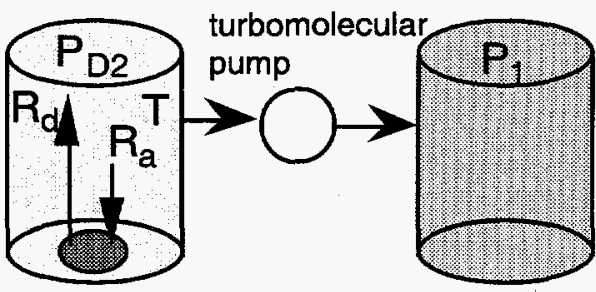

Fig. 3. A simple model of a pumped system.

Thus, we expect the deuterium concentration in the target of the pumped system to be reduced by a factor of $10^{5}$ relative to that of the unpumped system.

\section{Target Desorption Inlet System}

The flow schematic of the target desorption inlet system (TDIS) is shown in Fig. 4. The major components of the TDIS housed inside an insulated and temperature-controlled box are

- Three calibrated gas-storage vessels,

- A perforated foil molecular leak,

- A precision capacitance manometer ( 0 to 1 torr MKS Baratron),

- A cold cathode ionization gauge,

- A precision thermometer for the insulated box, and

- A circulating heater fan with thermostat. 
Other components located outside the insulated box are

- A crucible furnace with lifting mechanism and temperature readout,

- A liquid nitrogen trap to collect any condensable gases,

- A quartz thimble with a target loading and dropping mechanism,

- A turbomolecular pump for gas transfer,

- A capacitance manometer ( 0 to 1000 torr) for the pump-out line,

- Two gas purifiers as hydrogen gas scavenger pumps, and

- An oil-free dry high-vacuum pumping system consisting of a turbomolecular drag pump and a diaphragm backing pump.

With the inlet system under high vacuum, desorption is accomplished by lifting the furnace set at the desorption temperature to completely surround the quartz thimble. After the system reaches thermal equilibrium, the target is then dropped into a hot zone. The desorbed gas is passed through a liquid nitrogen trap, then transferred to the gas storage vessel. PVT measurements are made to determine the total amount of gas. Then the offgas is admitted to the mass spectrometer for isotopic analysis.

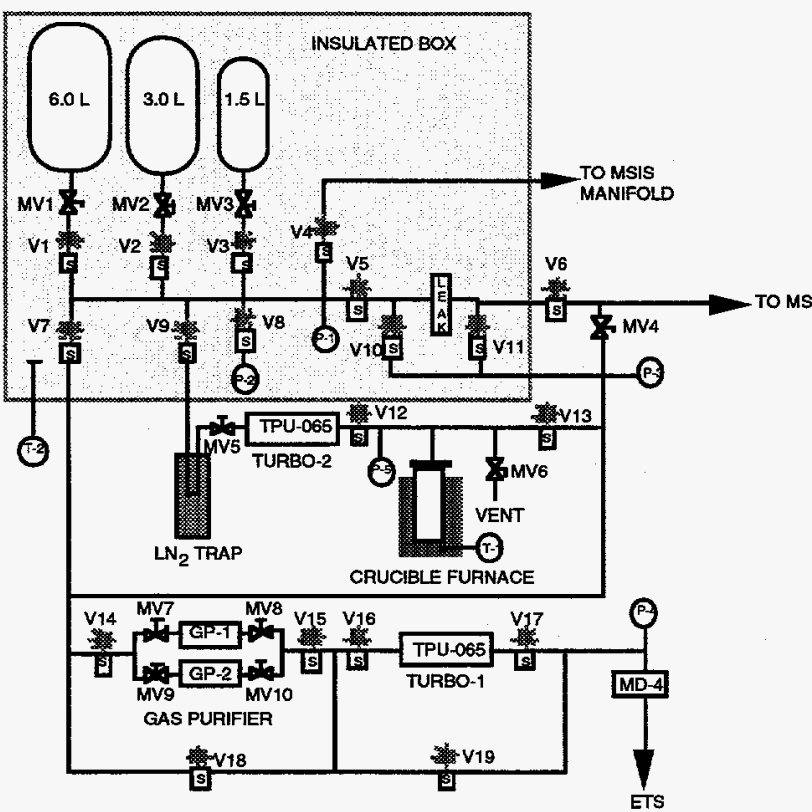

The inlet system valves can be controlled manually or by computer. The pumping system is capable of achieving a pressure of about $10^{-5}$ torr in less than 30 minutes to reduce the contribution of previously analyzed gases to a few parts per million (ppm) for the sample currently being analyzed. The amount of hydrogen isotopes scavenged in the gas purifiers can be recovered. All hydrogen isotopes going through the inlet system and mass spectrometer are exhausted to the effluent treatment system (ETS) from which future recovery is possible.

\section{Mass Spectrometer}

The Premier American Technology Corporation (PATCO) 6-90-GQN mass spectrometer is a singlefocusing instrument, 6 inches in radius, with a $90^{\circ}$ magnetic sector and double dispersion. It features a gas-tight ion source, high sensitivity, and high resolution. It has an adjustable source and image slit setting for resolving power in steps of 900,1200 , 2400 , and 4800 .

The ion-forming region and inner surface are passivated using Quantum Mechanics ${ }^{3}$. The ion source housing end is pumped with a $240-\mathrm{L} / \mathrm{s}$ turbomolecular pump backed by both a turbomolecular drag and an oil-free diaphragm pump.

The internal surfaces of the analyzer and detector housing are also passivated by Quantum Mechanics ${ }^{\circledR}$. Pumping is provided by a $50-\mathrm{L} / \mathrm{s}$ turbomolecular pump at the source end and a $25-\mathrm{L} / \mathrm{s}$ triode ion pump at the detector end.

The instrument is equipped with a retractable Faraday cup ion collector and a Channeltron-type electron multiplier to operate on a center beam axis.

Modern solid-state technology and fiber-optic electronics are used. The ion accelerating potential, ion lens voltages, emission regulator, and ion source temperature are designed for both manual and computer control.

Fig. 4. TDIS flow schematic. 


\section{EXPERIMENTAL DATA}

\section{Pressure Curve Characteristics of the Unpumped and Pumped System}

The qualitative difference between the unpumped and pumped systems is illustrated in Fig. 5 in the form of the time it takes for the system to reach equilibrium. In the unpumped inlet system, the offgas pressure slowly reaches equilibrium after 3 minutes of desorption. In the pumped system, the offgas is transferred and expanded into the gas-storage vessel, and the system reaches equilibrium in less than one minute.

After reaching equilibrium, both systems are then cooled down and pumped out, and their desorption efficiencies are measured. The results of measurements after being pumped down twice (shown in Fig. 6) indicated that in the unpumped system, a significant amount of gas remains in the target. By contrast, $99.6 \%$ of the deuterium comes off in the first step in the pumped system, and only $0.4 \%$ of the deuterium comes off in the second step. No deuterium is detected in the third step.

\section{Apparent Volume Calibration}

The volume of the gas is not static as a result of the presence of the turbomolecular pump. There is also a temperature gradient between the liquid nitrogen trap and the gas storage vessel. Therefore,

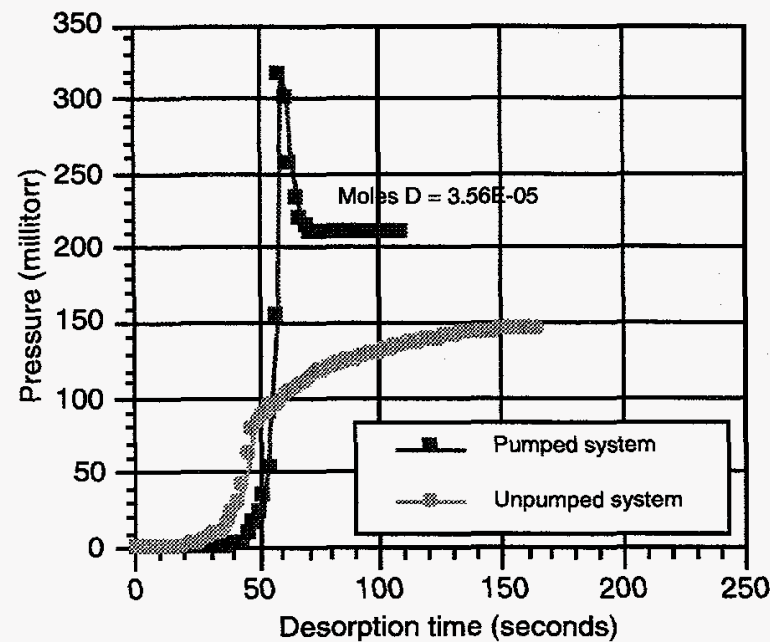

Fig. 5. Pressure curve characteristics of the pumped and unpumped systems.

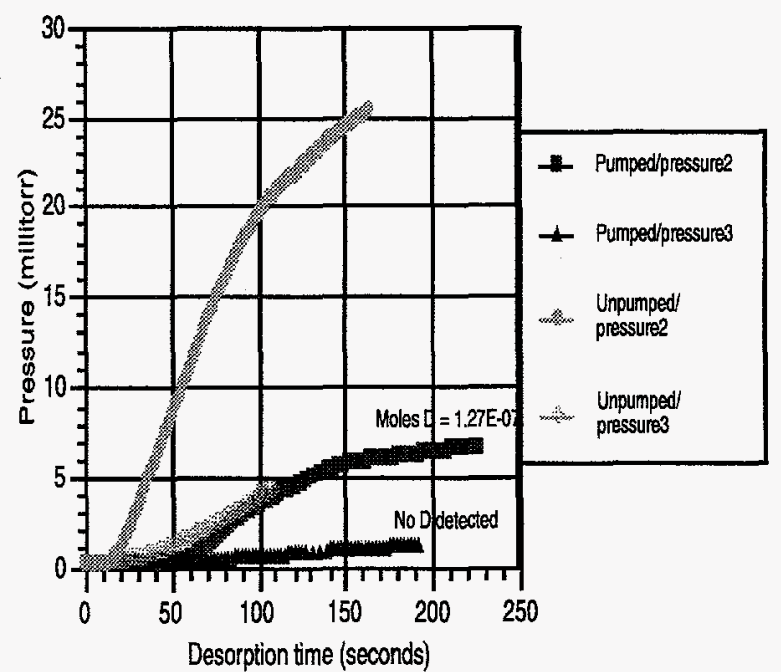

Fig. 6. Two repeated pump-down experiments illustrate the difference in desorption efficiency of the pumped and unpumped systems.

the apparent volume is calibrated with the turbomolecular pump turned on and the liquid nitrogen trap filled. The apparent volume is calibrated at the different turbomolecular pump outlet pressures as illustrated in Fig. 7. This pressure range is selected near the range of the gas pressure that is expected to come off from the target to be analyzed. The 1.5-L calibrated gas-storage vessel is filled with deuterium and subsequently expanded into different sections of the manifolds and volumes. The solid line indicates the best nonlinear curve fit for this set of data. The curve-fit equation is entered into the calculations and used to correct the apparent volume as a bias of the turbomolecular pump outlet pressure.

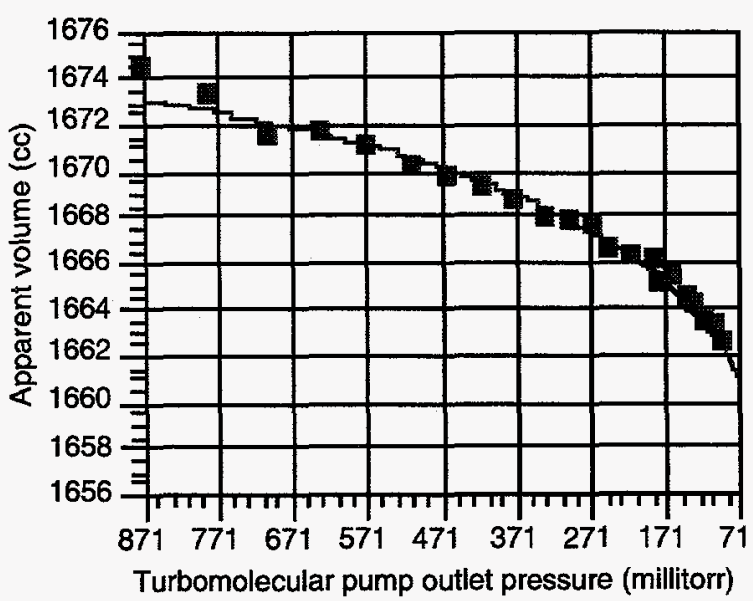

Fig. 7. Apparent volume as a bias of the turbomolecular pump outlet pressure. 


\section{Optimum Desorption Temperature and Time}

The optimum temperature and time are determined experimentally so that we can desorb all the gases in the shortest period of time without causing any erbium sublimation. Because the weight of the metal film varies somewhat from production run to production run, the targets with similar weights were chosen for this experiment. Figure 8 shows the desorption characteristics as a function of temperature. Desorption takes longer as the temperature decreases. Furthermore, the desorption efficiency is very low- $97 \%$ at $650^{\circ} \mathrm{C}$ and $98 \%$ at $750^{\circ} \mathrm{C}$. At $900^{\circ} \mathrm{C}$, more than $99.5 \%$ of the total amount of gas is released in the first step. For the case study of deuterated erbium targets, the optimum desorption temperature is $850^{\circ} \mathrm{C}$ to $900^{\circ} \mathrm{C}$, and the optimum desorption time is 2 to 3 minutes.

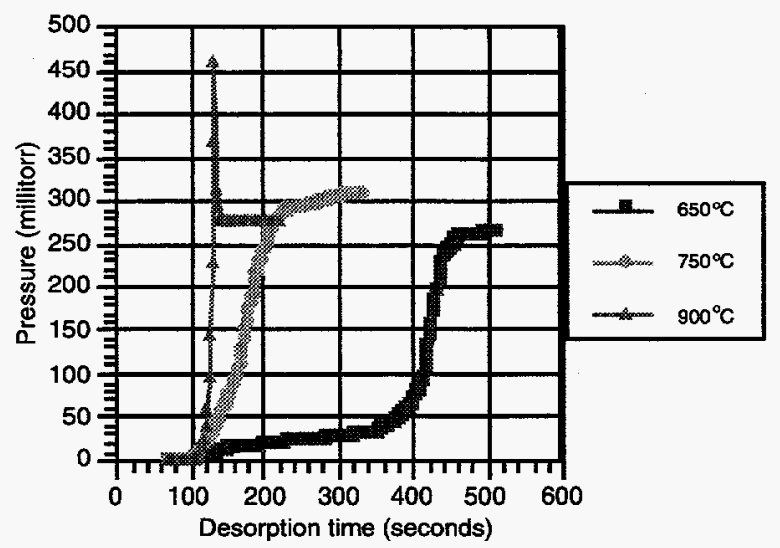

Fig. 8. The desorption characteristics as a function of temperature of the pumped system.

\section{GAS ANALYSIS RESULTS AND DISCUSSION}

Table 1 shows the gas analyses results of the total hydrogen-gas-to-erbium ratio (gas/Er) and the deuterium-to-erbium ratio (D/Er) of the deuteriated targets measured at different desorption times.

The total gas/Er ratios are compared based on the amount of erbium loaded on the targets by weight differences. ${ }^{4}$ The D/Er ratios are compared based on the weight differences and wet chemistry using isotope dilution and inductive coupled plasma mass spectrometry. ${ }^{5}$ This technique is another way to
Table 1. Gas/Er and D/Er ratio of deuterated targets at different desorption times.

\begin{tabular}{|c|c|c|c|c|c|}
\hline $\begin{array}{r}\text { Sample } \\
\text { ID }\end{array}$ & $\begin{array}{c}\text { Prebaked } \\
\text { temp } \\
\left({ }^{\circ} \mathrm{C}\right) / \text { time } \\
\text { (hh:mm) }\end{array}$ & $\begin{array}{c}\text { Desorption } \\
\text { temp } \\
\left({ }^{\circ} \mathrm{C}\right) / \text { time } \\
(\mathrm{mm}: \mathrm{ss})\end{array}$ & $\mathrm{Gas} / \mathrm{Er}^{\dagger}$ & $\mathrm{D} / \mathrm{Er}^{\ddagger}$ & $\mathrm{D} / \mathrm{Er}^{8}$ \\
\hline 1346 & $750 / 1: 25$ & $900 / 2: 00$ & 2.00 & 1.93 & 1.95 \\
\hline 1344 & $750 / 4: 30$ & $900 / 2: 00$ & 1.98 & 1.94 & 1.91 \\
\hline 1351 & $750 / 4: 00$ & $900 / 0: 53$ & 1.95 & 1.92 & 1.87 \\
\hline 1353 & $750 / 0: 25$ & $900 / 1: 00$ & 1.97 & 1.93 & 1.93 \\
\hline 1404 & $900 / 0: 30$ & $900 / 1: 00$ & 1.96 & 1.92 & 1.90 \\
\hline 1398 & $900 / 0: 15$ & 900/2:00 & 2.02 & 1.98 & 2.14 \\
\hline 1396 & $900 / 0: 50$ & $900: 1: 30$ & 2.04 & 2.00 & 1.91 \\
\hline $1386 *$ & 900/0:40 & 900/3:00 & 2.05 & 1.98 & 2.07 \\
\hline $1387^{*}$ & $900 / 0: 30$ & 900/2:00 & 2.06 & 2.00 & 2.06 \\
\hline $1388^{*}$ & 900/1:00 & $900 / 3: 00$ & 2.06 & 2.01 & 2.06 \\
\hline $1391 *$ & $900 / 1: 00$ & $900 / 3: 00$ & 2.06 & 2.02 & 2.11 \\
\hline
\end{tabular}

*Samples with two additional repeated pump-down desorption steps for a total of 6 minutes.

${ }^{\dagger} \mathrm{Gas} / \mathrm{Er}$ ratio based on the Er weight differences.

${ }^{\ddagger} \mathrm{D} / \mathrm{Er}$ ratio based on the Er weight differences.

${ }^{\S} \mathrm{D} / \mathrm{Er}$ ratio based on the $\mathrm{Er}$ weight determined by wet chemistry technique.

verify if the target is desorbed under the optimum operating conditions.

The gas/Er ratios are close to the stoichiometric ratio of an erbium dihydride system. The variation in the gas/Er and $\mathrm{D} / \mathrm{Er}$ ratio measurement depends not only on the variation of the desorption parameters but also on the target loading parameters and Er weight measurements. The variations resulting from the latter two are beyond the discussion of this paper.

At the same temperature of $900^{\circ} \mathrm{C}$, the $\mathrm{D} / \mathrm{Er}$ ratios are higher with 2 to 3 minutes of desorption (samples 1396, 1398, 1386, 1387, 1388, and 1391) than those with 1 to 2 minutes.

For the last four samples (1386 to 1391), the D/Er ratios based on Er wet chemistry techniques are much higher than the results obtained from the $\mathrm{Er}$ weight differences. This indicates that some of erbium may be sublimed because of the longer heat exposure in the two additional desorption steps for a total of 6 minutes. The purpose of the two additional steps is to verify the complete desorption of the gas. 
Table 2 shows the gas analyses results of the gas/Er of the tritiated erbium targets. At the same desorption time of 3 minutes, the total gas/Er ratio increases as the desorption temperature increases. However, it is closer to a stoichiometric ratio at a desorption temperature of $900^{\circ} \mathrm{C}$.

Table 2. Gas/Er ratio of tritiated targets at different desorption temperatures and times.

\begin{tabular}{|l|c|c|c|c|}
\hline Sample ID & $\begin{array}{c}\text { Prebaked } \\
\text { temp }\left({ }^{\circ} \mathrm{C}\right) / \\
\text { time } \\
(\mathrm{hh}: \mathrm{mm})\end{array}$ & $\begin{array}{c}\text { Desorption } \\
\text { temp }\left({ }^{\circ} \mathrm{C}\right) / \\
\text { time } \\
(\mathrm{mm}: \mathrm{ss})\end{array}$ & $\begin{array}{c}\text { Gas/ } \\
\mathrm{Er}^{*}\end{array}$ & $\begin{array}{c}\text { Gas/ } \\
\mathrm{Er}^{\dagger}\end{array}$ \\
\hline 1224 & $850 / 1: 30$ & $850 / 2: 20$ & 1.95 & - \\
\hline 9609012 & $850 / 1: 00$ & $850 / 3: 00$ & - & 1.92 \\
\hline 9609128 & $850 / 3: 00$ & $850 / 3: 00$ & - & 1.96 \\
\hline 1253 & $875 / 1: 30$ & $875 / 3: 00$ & 1.93 & 1.96 \\
\hline 9613135 & $875 / 1: 30$ & $875 / 3: 00$ & - & 1.90 \\
\hline 1235 & $875 / 2: 00$ & $875 / 3: 00$ & 1.91 & 1.91 \\
\hline 9609164 & $875 / 3: 00$ & $875 / 3: 00$ & - & 1.86 \\
\hline 1233 & $875 / 1: 30$ & $875 / 2: 30$ & 1.93 & 1.93 \\
\hline 9609151 & $875 / 1: 00$ & $875 / 3: 00$ & - & 1.92 \\
\hline 1225 & $900 / 1: 25$ & $900 / 3: 00$ & 2.00 & - \\
\hline
\end{tabular}

* Gas/Er ratio based on the Er weight differences.

${ }^{\dagger} \mathrm{Gas} / \mathrm{Er}$ ratio based on the Er weight determined by wet chemistry techniques.

\section{CONCLUSION}

The modified TDIS has performed very well. Its complete desorption, rapidity, high sensitivity, and versatility improves measurement accuracy, reduces the measurement time, and can be readily adapted to the analysis of other metal hydride targets.

For the case study of deuterated and tritiated erbium targets, the optimum desorption temperature is $850^{\circ} \mathrm{C}$ to $900^{\circ} \mathrm{C}$, and the optimum desorption time is 2 to 3 minutes.

We have observed a high background contribution from the quartz thimble at the operating temperature. In addition, measurement precision is improved as the quartz thimble ages. The gas/Er and D/Er ratio measurements are very consistent between targets with great variation in their metal weights. A precision of $2 \%$ or better is achieved in most cases.

\section{REFERENCES}

1. R. S. Kunhardt, "Thin Film Analysis-MAT 251 Mass Spectrometer," UNC, GEND Chemistry Laboratory report CHM 5220-1, May 22, 1991.

2. T. K. Merhoff, "PVT Calibration Technique for Mass Spectrometer Inlet Systems," UNC, GEND Chemistry Laboratory report CHM 81-119, May $21,1981$.

3. J. Brangan and S. Thornberg, "Deuterated Target Gas Analysis," progress report presented at Los Alamos-Sandia National Laboratory project-related team meeting, November 2, 1995.

4. LMSC's Neutron Generator Operations Tube Manufacturing Unit Data Record, private communication, September 1995.

5. S. Rodriguez, H. Bach, C. Brink, G. Havrilla, F. Steinkruger, S. Black, and J. Olivares, "High Precision and Accuracy Erbium Analysis," Poster session presented at the Los Alamos National Laboratory Chemical Science and Technology Division Review meeting, June 20, 1996. 